\title{
Angioplastia Transluminal Coronariana em Portador de Púrpura Trombocitopênica Idiopática
}

\author{
Percutaneous Trans/uminal Coronary Angioplasty in a Patient with Idiopathic \\ Thrombocytopenic Purpura
}

\author{
Luis Gustavo M. Marques, Murillo Kenji Furukawa, Thenyson Pereira Leitão, \\ José Luis A. Quiñones, Fernando César de Queiroz, Rogerio Felippe Tiossi, \\ Virgílio Ribeiro Franco Jr, Carlos Eduardo M. Domingues, Decio Salvadori Jr \\ São Paulo, SP
}

A associação entre a doença arterial coronariana e a púrpura trombocitopênica é rara e implica em algumas dificuldades quando a revascularização se faz necessária. Relatamos um caso dessa associação, com importante comprometimento coronariano, tratado percutaneamente com o implante de stent.
The association between coronary heart disease and thrombocytopenic purpura is rare and poses some difficulties when myocardial revascularization is necessary. We report a case with this association and significant coronary impairment, which was percutaneously treated with stent implantation.
A púrpura trombocitopênica Idiopática, também conhecida como doença de Werlhof, tem incidência rara na população geral. Ocorre em todas as faixas etárias, nas formas aguda e crônica. As crianças apresentam, predominantemente, a forma aguda, com o acometimento equalizado de ambos os sexos. Já os adultos apresentam, principalmente, a forma crônica, com maior incidência no sexo feminino $(3: 1)^{1}$. Ambas as formas têm como fisiopatologia a produção de anticorpos anti-plaquetas, usualmente da classe IgG, levando à destruição acelerada das plaquetas pelo sistema retículo-endotelial.

A associação da púrpura com a doença arterial coronariana também é rara. Relatamos um caso de associação entre essas duas doenças, tratado, percutaneamente, com o implante de dois stents intracoronarianos.

\section{Relato do caso}

Homem de 54 anos, relatando dor precordial típica associada a dispnéia, com início há cerca de 4 meses, associada a médios esforços, com piora rápida e, a uma semana da internação, apresentando-se com dor a pequenos esforços e mesmo em repouso.

Como antecedentes relatava ser hipertenso e diabético, em uso de captopril - $75 \mathrm{mg} / \mathrm{d}$ e de glibenclamida - $10 \mathrm{mg} / \mathrm{d}$. Referia também ser portador de púrpura trombocitopênica, sendo acompanhado, regularmente, por hematologista de sua cidade e fazendo uso de prednisona - $60 \mathrm{mg} / \mathrm{d}$, não apresentando sangramentos

Real e Benemérita Associação Portuguesa de Beneficência - São Paulo Endereço para correspondência: Murillo Kenji Furukawa Rua Haddock Lobo, 1459/102 - Cep 01414-003 - São Paulo, SP E-mail: mkenji@cardiol.br

Enviado em 30/04/2004 - Aceito em 03/08/2004 patológicos nos últimos meses. Negava antecedentes familiares de doença arterial coronariana, tabagismo ou hipercolesterolemia.

Ainda em sua cidade, foi submetido a cinecoronariografia que mostrou circulação coronariana de dominância esquerda com lesões graves nas artérias descendente anterior (DA), circunflexa (CX) e no ramo primeiro marginal esquerdo (ME-1) (fig. 1). Notadamente, apresentava ainda origem anômala da artéria coronária direita $(C D)$ no terço proximal da $D A$, junto à lesão dessa artéria, estando a própria CD com lesão importante na sua origem.

0 paciente foi encaminhado para a realização de cirurgia de revascularização do miocárdio. À admissão, o hemograma mostrou $\mathrm{Hb}$ de $10,4 \mathrm{~g} / \mathrm{dl}$, $\mathrm{Ht}$ de $28,8 \%$ e plaquetopenia acentuada com $8.000 \mathrm{plaq} / \mathrm{mm}^{3}$. Não havia outras alterações laboratoriais dignas de nota.

O caso foi avaliado pela hematologia, que tratou, primeiramente a anemia e a plaquetopenia, iniciando também a administração de metil-prednisolona e imunoglobulina e orientando a realização da cirurgia, somente após a obtenção de pelo menos 90.000 $\mathrm{plaq} / \mathrm{mm}^{3}$. Entretanto, mesmo após várias transfusões ao longo de 20 dias, atingiu-se um máximo de 86.000 plaq $/ \mathrm{mm}^{3}$ (fig. 2). Nesse período houve piora importante do quadro anginoso, sendo necessária a transferência para Unidade Coronariana e uso de altas doses de nitroglicerina EV (Tridil ${ }^{\circledR}$ ). Como não obtivesse meIhora da concentração de plaquetas, foi solicitada avaliação da cardiologia intervencionista com vistas à realização de angioplastia transluminal coronariana.

O eletrocardiograma prévio mostrava ritmo sinusal, com alterações difusas e inespecíficas da repolarização ventricular.

O paciente foi então submetido a dissecção da artérial braquial direita, decorrida sem incidentes. No dia do procedimento o paciente apresentava uma contagem de $15.000 \mathrm{plaq} / \mathrm{mm}^{3}$. Após a dissecção da artéria braquial foi administrada heparina EV na dose de $70 \mathrm{U} / \mathrm{Kg}$ e utilizado um cateter-guia modelo Wiseguide ${ }^{\mathrm{TM}} \mathrm{FL}$ 

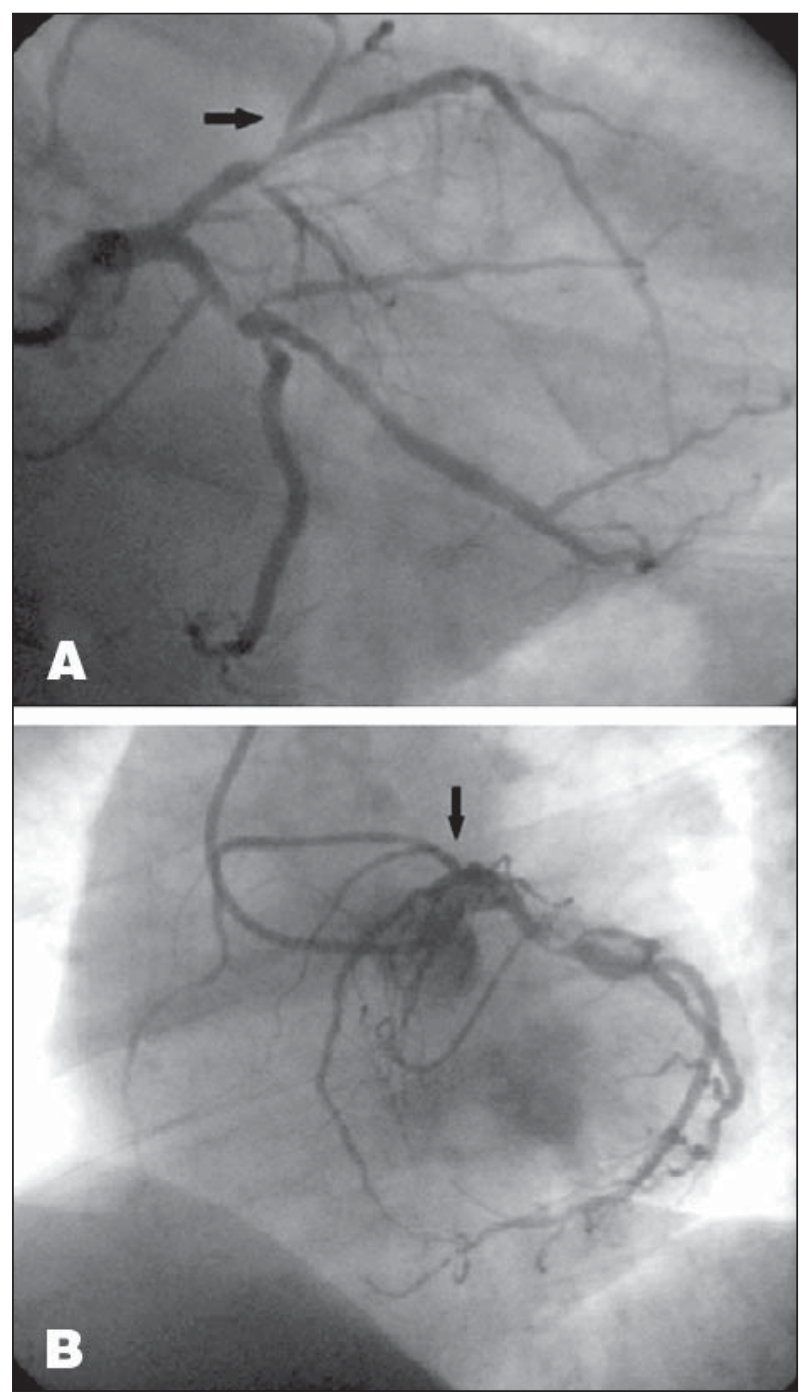

Fig. 1 - A - vista em OAD caudal da CE; B - vista em OAE da CE. As setas mostram a origem anômala da $C D$.

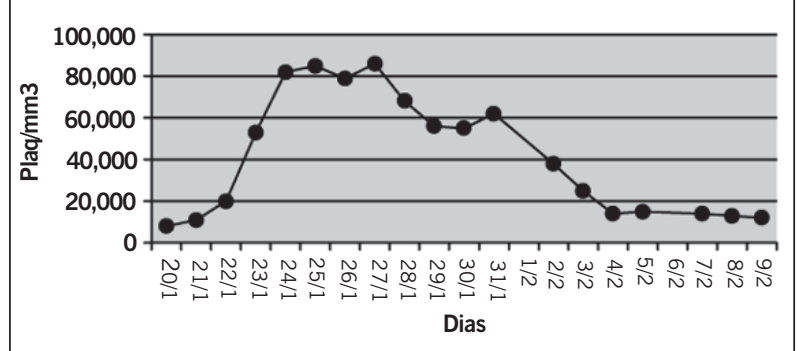

Fig. 2 - Evolução da concentração de plaquetas durante a internação.

3,5 SH - 6F e uma corda-guia 0,014" - $182 \mathrm{~cm}$ modelo Choice PT Extra Support ${ }^{T M}$ (ambos da Boston Scientific - SCIMED). Inicialmente, abordou-se a lesão da artéria $C X$, ultrapassada sem dificuldades pela corda-guia. A seguir, implantou-se, de forma direta, um stent modelo Driver-RX 3,5×15 mm (Medtronic AVE), liberado com 12 atm. Redirecionou-se, então, a corda-guia para a artéria DA, novamente passando-se a lesão sem grandes dificuldades. Implantou-se, também de forma direta, outro stent modelo Driver-RX, desta vez 3,5x18 mm, com 14 atm, obtendo-se bom resultado final (fig. 3). A despeito da $C D$ originar-se de forma sua vez, originar-se junto à lesão da $C X$, não houve oclusão de nenhum deles. Notou-se uma diminuição importante do fluxo na CD (TIMI I) sem, entretanto, a ocorrência de dor precordial ou instabilidade hemodinâmica.

Após a constatação de resultado satisfatório em ambas as artérias tratadas, encerrou-se o procedimento. A artéria braquial foi suturada com um fio prolene 6-0, não se observando a ocorrência posterior de sangramentos ou hematomas.

No seguimento intra-hospitalar, o paciente apresentou boa evolução, com melhora do quadro anginoso. A CKMB pré-ATC era de $42 \mathrm{U} / \mathrm{l}$, havendo normalização já nas primeiras 24h (fig. 4). 0 eletrocardiograma pós-ATC não mostrou alterações em relação ao pré-ATC.

Embora o grupo de hematologia não aconselhasse a utilização de anti-plaquetários, optou-se, em comum acordo, administrar enoxaparina por via subcutânea na dose de $1 \mathrm{mg} / \mathrm{kg} /$ dia durante o período hospitalar. Entretanto, nas primeiras 24h após o início da administração da droga houve 3 episódios de epistaxe, e nova contagem de plaquetas revelou a concentração de $11.000 / \mathrm{mm}^{3}$. A enoxaparina foi suspensa e o paciente mantido sem qualquer tipo de heparina ou anti-plaquetário. Dois dias após, recebeu alta hospitalar, evoluindo sem precordialgia ou sangramentos. Recebeu orientação de controle rigoroso com o hematologista de sua cidade, sendo-Ihe solicitado a liberação de pelo menos um antiagregante, assim que se verificasse valores de concentração plaquetária que permitissem a utilização de tais drogas.

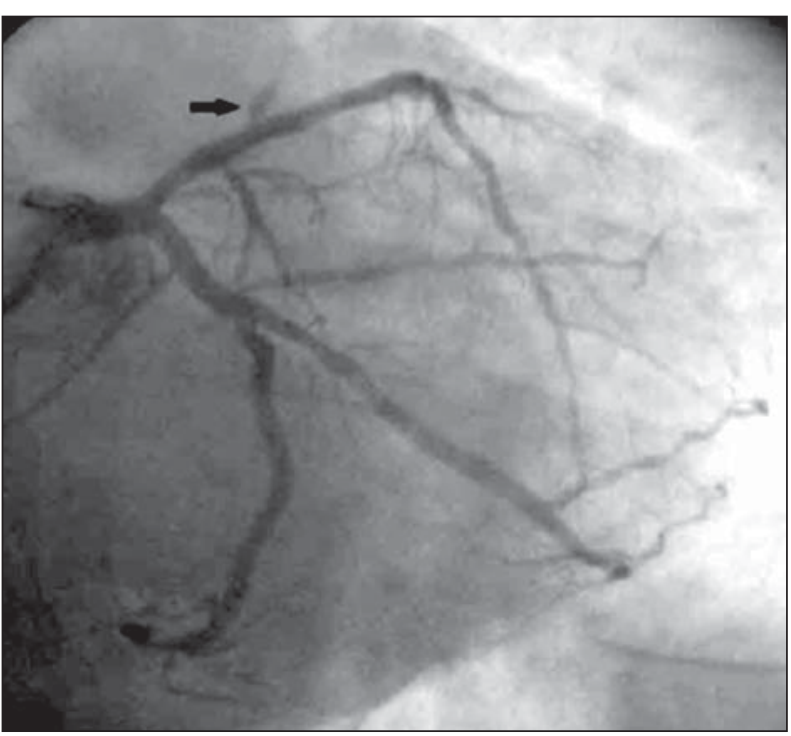

Fig. 3 - CE em OAD caudal pós-ATC; a seta mostra a diminuição do fluxo na CD.

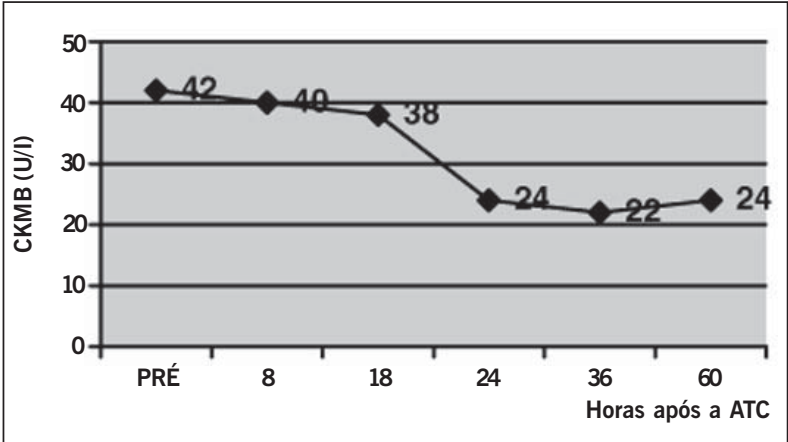

Fig. 4 - Curva dos valores de CKMB (VN: até 25 U/I). 
Em reavaliação telefônica recente, 4 meses após o procedimento, o paciente apresenta-se sem angina. Durante esse período não iniciou o uso de qualquer droga antiplaquetária, por recomendação de seu hematologista, devido à persistência da plaquetopenia acentuada e atingiu um máximo de $20.000 \mathrm{plq} / \mathrm{mm}^{3}$, sendo a maioria dos valores inferiores a 10.000; última contagem em 22/06/04 mostrava $3.000 \mathrm{plq} / \mathrm{mm}^{3}$. Continua sob uso de corticóides e imunoglobulinas, aguardando melhora para ser submetido a esplenectomia. Faz uso ainda de captopril - $50 \mathrm{mg} / \mathrm{dl}$, atenolol - $100 \mathrm{mg} / \mathrm{d}$ e mononitrato de isossorbida $-30 \mathrm{mg} / \mathrm{d}$.

\section{Discussão}

A púrpura trombocitopênica idiopática tem incidência rara. Levantamento realizado no Japão mostra uma prevalência de 15,5 casos ao ano para cada 100.000 indivíduos $^{2}$. A associação entre a doença arterial coronariana e a púrpura também é rara, e relatos na literatura mostram boa evolução desse quadro, quando é possível se elevar, adequadamente, a concetração plaquetária e submeter o paciente a revascularização cirúrgica ${ }^{3,4}$.

No caso descrito, observamos uma forma agressiva dessa doença, associada a grave doença coronariana obstrutiva. Uma possível explicação para a acentuada plaquetopenia, com inexpressiva resposta terapêutica, pode ser a concomitância de importantes níveis de estresse, gerados pela precordialgia e pela iminência de uma cirugia de grande porte.

Embora dispuséssemos de sistemas para a hemostasia do local de punção femoral (do tipo Perclose - AbbotVascular Devices), consideramos a possibilidade da ocorrência de acidentes durante a punção da artéria, que acarretaria, diante de níveis tão baixos de concentração plaquetária, complicações hemorrágicas antes mesmo de se iniciar o procedimento intervencionista. Não utilizamos a abordagem radial por não dispormos do material necessário, além da falta de experiência de nosso grupo com essa técnica. Optamos, então, pela dissecção da artéria braquial direita, o que permitiu visualização direta da artéria e melhor controle da hemostasia. Embora o paciente já tivesse sido submetido previamente a cinecoronariografia por essa técnica, observamos que a artéria braquial encontrava-se facilmente palpável acima da primeira incisão e sem diminuição do pulso radial.
Angioplastia Transluminal Coronariana em Portador de Púrpura Trombocitopênica Idiopática

Apesar do paciente apresentar-se com plaquetopenia importante, consideramos prudente a utilização, pelo menos, da enoxaparina, já que foram implantados dois stents na DA e na CX, que por sua vez eram dominantes. Entretanto, a ocorrência do sangramento (epistaxe) impôs a retirada dessa droga, e o paciente foi mantido sem qualquer tipo de heparina ou antiplaquetário.

Observação importante deve ser feita com relação à introdução de algum antiagregante plaquetário assim que possível. Fuchi e cols. ${ }^{5}$ realizaram uma angioplastia transluminal coronariana primária em portador de púrpura trombocitopênica idiopática e com quadro de infarto agudo do miocárdio, onde se observou suboclusão da artéria DA no seu terço proximal e outra lesão, de $50 \%$, no terço médio. Embora a angioplastia transluminal coronariana da lesão proximal tenha sido realizada com sucesso, a paciente desenvolveu, $9 \mathrm{~h}$ depois, novo episódio de infarto agudo do miocárdio com oclusão no terço médio da DA junto à lesão de $50 \%$. Os autores consideraram a possibilidade de ter havido lesão iatrogênica pela corda-guia durante a primeira angioplastia transluminal coronariana, associada a um aumento da viscosidade sangüínea causado pelo contraste e pela reposição das plaquetas. Ressaltaram a importância de se realizar as transfusões com cuidado, atendo-se ao mínimo necessário, a fim de evitar complicações trombogênicas após a angioplastia transluminal coronariana nesses portadores de púrpura trombocitopênica.

No nosso caso foi solicitado acompanhamento rigoroso pelo hemotologista, com a introdução de pelo menos alguma droga antiagregante assim que se obtivesse concentrações aceitáveis de plaquetas, mantendo-se por um período mínimo suficiente para permitir a endotelização dos stents.

Nos casos de associação entre a púrpura trombocitopênica e a doença arterial coronariana, a angioplastia transluminal coronariana com o implante de stents apresenta-se como opção terapêutica atraente. Além de constituir método, comprovadamente eficaz, a introdução de técnicas mais recentes, notadamente a abordagem radial, torna o procedimento mais seguro para pacientes com essa associação, expondo-os a trauma mínimo e com excelente controle da hemostasia. Como precaução, chamamos a atenção para o controle hematológico rigoroso, de forma a balancear os níveis de concentração plaquetária e o uso dos antiagregantes, tendo como objetivo evitar a ocorrência de complicações trombóticas.

\section{Referências}

1. Burns TR, Saleem A. Idiopathic thrombocytopenic purpura. Am J Med 1983; 75: 1001-7.

2. Oda K, Fujimura K, Kuramoto A. ITP (Idiopathic Thrombocytopenic Purpura). Nitijo Shinryo To Ketsueki 1995; 5: 25-30.

3. Mathew TC, Vasudevan R, Leb L et al. Coronary artery bypass grafting in immune thrombocytopenic purpura. Ann Thorac Surg 1997; 64: 1059-62.
4. Thompson LD, Cohen AJ, Edwards FH, Barry MJ. Coronary artery bypass in idiopathic thrombocytopenia without splenectomy. Ann Thorac Surg 1989;48:721-2.

5. Fuchi T, Kondo T, Sase K et al. Primary Percutaneous Transluminal Coronary Angioplasty Performed for Acute Myocardial Infarction in a Patient With Idiopathic Trombocytopenic Purpura. Jpn Circ J 1999;63:133-6. 